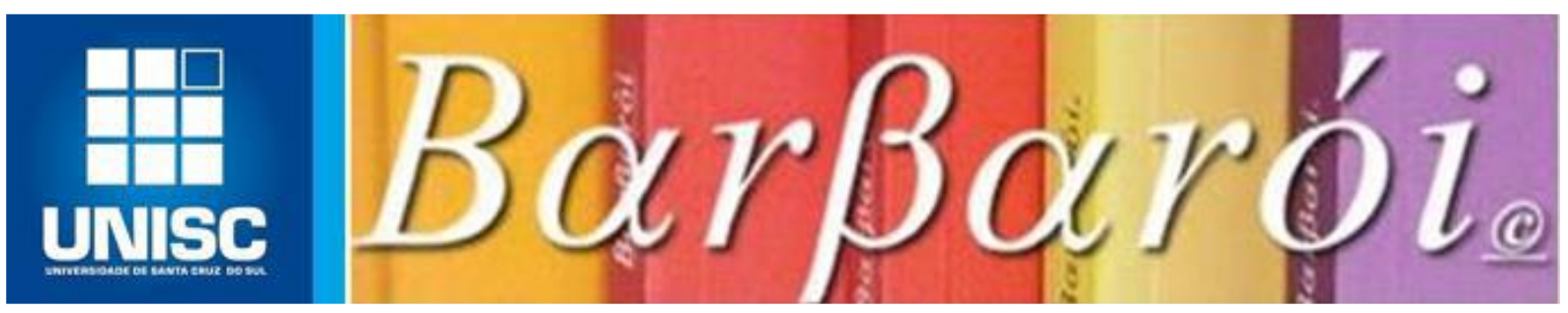

\title{
PERSPECTIVAS DE ADOLESCENTES SOBRE A PRÁTICA DE CUIDADO DOS IRMÃOS: UM ESTUDO QUALITATIVO
}

\author{
DOI: http://dx.doi.org/10.17058/barbaroi.v0i58.6538

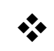 \\ Gabriella Koltermann \\ Universidade Federal do Rio Grande do Sul - UFRGS - Brasil \\ Meiridiane Domingues de Deus \\ Universidade Federal de Santa Catarina - UFSC - Brasil \\ Samara Silva dos Santos \\ Universidade Federal de Santa Maria - UFSM - Brasil \\ $*$
}

\section{Resumo}

Este estudo buscou investigar as perspectivas de adolescentes sobre a prática de cuidado dos irmãos. A amostra foi composta por seis adolescentes de ambos os sexos, com idades entre 11 e 19 anos. Os dados foram coletados por meio de entrevistas semiestruturadas e as informações foram submetidas à análise de conteúdo. Ressalta-se a importância das atividades desempenhadas pelos adolescentes no contexto familiar para o sustento financeiro da família. A percepção do cuidador sobre o cuidado dispensado aos irmãos abarcou aspectos positivos e negativos, sendo também considerada uma prática normal e rotineira. Dentre os pontos positivos, constatou-se transmitir algo de bom aos irmãos, os desafios e facilidades na realização de atividades de cuidado com os irmãos menores. A prática do cuidado pode contribuir para o sentimento de importância no contexto familiar, como também ser percebida como uma falha das mães no cumprimento de suas responsabilidades. As percepções negativas referiram-se à limitação dos tempos de lazer, à rotina estressante e à mediação de brigas entre os irmãos menores. Evidencia-se a importância de analisar o que essa prática representa para os adolescentes cuidadores, já que esta pode estar associada a sentimentos de desconforto, influenciar o bem-estar, bem como limitar a realização de atividades de lazer.

Palavras-chave: Irmãos; Cuidadores; Adolescente.

\section{Introdução}

O papel de cuidador de uma criança envolve muitas habilidades e responsabilidades. As figuras parentais são fontes comuns de estimulação para o desenvolvimento infantil por meio de momentos de interação verbal e não verbal entre pais, mães e filhos(as) (por 
exemplo, alimentação, banho, brincadeiras e ajuda nas tarefas escolares). Portanto, pais e mães estão bastante envolvidos nos processos de aprendizagem da criança, no que se refere aos aspectos emocionais, sociais e cognitivos (LAM; MCHALE; CROUTER, 2012; MERMELSHTINE, 2017; TUCKER; UPDEGRAFF, 2009).

Entretanto, em famílias brasileiras, principalmente de camadas populares, majoritariamente compostas por pessoas negras, mestiças e indígenas, numerosas, com configuração monoparental e baixo suporte social (inclusive indisponibilidade de vagas em instituições de ensino infantil), a função de cuidado da criança é, muitas vezes, atribuída ao seu irmão. Esses irmãos (crianças e adolescentes em sua maior parte) tornam-se corresponsáveis por crianças mais novas, realizando muitas das atividades comumente atribuídas às figuras parentais (MACMAHON; LUTHAR, 2007; POLETTO; WAGNER; KOLLER, 2004; SILVA; TOKUMARU, 2008; RUIZ-CASARES; HEYMANN, 2009). Por isso, é relevante que estudos analisem as experiências do irmão cuidador e a percepção destes sobre as atividades de cuidado (EAST, 2010). Até o momento, a literatura carece de estudos que explorem a perspectiva desses adolescentes que, muitas vezes, assumem mesmo que em parte os cuidados parentais tão importantes para o seu desenvolvimento e o de seus irmãos.

"Cuidados com os irmãos" (do inglês sibling caretaking) incluem todos os tipos de socialização, treinamento e responsabilidades rotineiras que uma criança ou adolescente assume pelos adultos significativos e responsáveis por essas tarefas. Essas atividades variam desde o cuidado completo e independente em tempo integral até o desempenho de tarefas específicas (WEISNER; GALLIMORE, 1977).

Essa relação de cuidado formal pode estar associada a resultados positivos e negativos para o desenvolvimento tanto daqueles que cuidam quanto daqueles que recebem cuidados. A prática de cuidar dos irmãos pode despertar nos adolescentes sentimentos positivos, ao possibilitar que eles se sintam orgulhosos em ajudar nas atividades relativas aos cuidados dos irmãos, além de promover o aprendizado do ato de cuidar, ter o reconhecimento de outra pessoa e poder realizar atividades semelhantes às funções maternas (DALHBLOM et al., 2009).

Por outro lado, as tarefas domésticas conceituadas como "ajuda", que incluem o cuidado dos filhos, podem vir acompanhadas de altas demandas físicas e psicológicas por quem as desempenha. Um estudo com adolescentes cambojanos indicou níveis aumentados de sintomas depressivos entre meninos que cuidavam dos irmãos, bem como baixo desempenho escolar e probabilidade percebida de abandono escolar. Entre as meninas, havia altos níveis de 
sintomas depressivos associados ao cuidado dos irmãos, bem como baixo desempenho escolar (YI et al., 2012). Assim, além de implicações negativas para o aproveitamento e retenção escolar, a responsabilidade de exercer o cuidado de outros familiares, o isolamento e a falta de apoio decorrentes dessa condição também podem causar danos à saúde física e mental de adolescentes, tanto meninas quanto meninos (SMYTH; BLAXLAND; CASS, 2011; YI et al., 2012). O escasso tempo para atividades de lazer também estaria relacionado às tarefas de cuidar dos irmãos (DELLAZZANA, 2008).

Partindo dessas perspectivas em relação ao cuidado dos irmãos, optamos por utilizar o modelo de sistemas ecológicos de Bronfenbrenner (1979), o qual consiste em quatro níveis ambientais - o microssistema, o mesossistema, o exossistema e o macrossistema - com cada nível interagindo entre si e impactando de forma diferente o desenvolvimento de cada pessoa. De acordo com Bronfenbrenner (1979), o microssistema envolve o ambiente imediato com o qual a criança ou o adolescente interage intimamente, como bairro, sala de aula e ambiente familiar. Exemplos são as interações entre irmãos típicas do microssistema familiar em que os indivíduos se encontram. Já o mesossistema neste contexto, refere-se à integração entre família e escola dos adolescentes; e o exossistema refere-se ao trabalho dos pais, mães, e/ou cuidadores; e o macrossistema, as leis e as políticas públicas que se relacionam a realidade dos adolescentes (ONWUEGBUZIE; COLLINS; FRELS, 2013).

Considerando o número expressivo de famílias que incitam o cuidado das crianças aos irmãos mais velhos diante das condições existentes (referentes ao exossistema e ao macrossistema), é importante destacar as práticas e as perspectivas dos irmãos cuidadores. De forma geral, os estudos referentes ao relacionamento fraterno no contexto brasileiro ainda são escassos e é importante considerar as características e variadas configurações e dinâmicas familiares existentes no Brasil (PEREIRA; PICCININI; LOPES, 2019). De fato, conforme comentado anteriormente, pouco se sabe sobre o cuidado dos irmãos nas famílias contemporâneas (WIKLE; JENSEN; HOAGLAND, 2018), principalmente em países em desenvolvimento (YI et al., 2012), como o Brasil. Portanto, este estudo tem o objetivo de abordar qualitativamente as atividades e as perspectivas de adolescentes sobre a prática de cuidado aos seus irmãos no contexto familiar. 


\section{Método}

\section{Participantes}

Participaram do estudo seis adolescentes, de ambos os sexos (4 meninas e 2 meninos), com idades entre 11 e 19 anos (Média de idade = 16 anos), que realizavam cuidados recorrentes para os irmãos. A escolaridade da amostra variou da $4^{\mathrm{a}}$ série do ensino fundamental ao ensino médio incompleto. $\mathrm{O}$ número de irmãos por participante variou de 2 a 7. O número de moradores na mesma casa variou entre 4 e 9 pessoas. A composição familiar foi caracterizada por duas famílias constituídas por pai, mãe e filhos; três formadas pela mãe e seus filhos e uma formada por padrasto, filhos e enteada. Essas características da amostra são detalhadas na Tabela 1 abaixo. Os nomes dos participantes não serão mencionados, assim optou-se por adotar códigos como P1, P2, P3, P4, P5 e P6, a fim de resguardar o sigilo da sua identidade. Os nomes citados pelos participantes também serão suprimidos.

Quadro 1. Informações sobre características familiares da amostra

\begin{tabular}{|c|c|c|c|c|c|c|}
\hline & $\mathrm{P} 1$ & $\mathrm{P} 2$ & P3 & $\mathrm{P} 4$ & P5 & P6 \\
\hline Idade & 18 anos & 11 anos & 14 anos & 14 anos & 19 anos & 17 anos \\
\hline Escolaridade & $\begin{array}{l}\text { Ensino Médio } \\
\text { Incompleto }\end{array}$ & $\begin{array}{l}\text { Ensino } \\
\text { Fundamenta } \\
1 \text { incompleto }\end{array}$ & $\begin{array}{l}\text { Ensino } \\
\text { Fundamenta } \\
1 \text { incompleto }\end{array}$ & $\begin{array}{c}\text { Ensino } \\
\text { Fundamenta } \\
1 \text { incompleto }\end{array}$ & $\begin{array}{l}\text { Ensino } \\
\text { Fundamenta } \\
\text { 1 Incompleto }\end{array}$ & $\begin{array}{c}\text { Ensino } \\
\text { Médio } \\
\text { Incompleto }\end{array}$ \\
\hline $\mathrm{N}^{\mathrm{o}}$ de irmãos & 6 & 2 & 2 & 3 & 4 & 7 \\
\hline $\begin{array}{l}\text { Idade dos } \\
\text { irmãos }\end{array}$ & $\begin{array}{c}9 \\
10,10,15,22,2 \\
5 \text { anos }\end{array}$ & 7 e 8 anos & 2 e 10 anos & $\begin{array}{c}8 \text { meses, } 3 \text { e } \\
8 \text { anos }\end{array}$ & $\begin{array}{l}6 \text { meses, } 5, \\
12 \text { e } 15 \text { anos }\end{array}$ & $\begin{array}{l}4,7,9,10,11, \\
20 \text { e } 23 \text { anos }\end{array}$ \\
\hline $\begin{array}{c}\mathrm{N}^{\circ} \text { de pessoas } \\
\text { na casa }\end{array}$ & 9 & 4 & 4 & 5 & 7 & 9 \\
\hline $\begin{array}{l}\text { Composição } \\
\text { familiar }\end{array}$ & $\begin{array}{l}\text { Pai, Mãe e } \\
\quad \text { filhos }\end{array}$ & $\begin{array}{c}\text { Padrasto, } \\
\text { filhos e } \\
\text { enteada }\end{array}$ & Mãe e filhos & $\begin{array}{l}\text { Pai, Mãe e } \\
\text { filhos }\end{array}$ & Mãe e filhos & Mãe e filhos \\
\hline $\begin{array}{l}\text { Ocupação } \\
\text { do(s) } \\
\text { responsável } \\
\text { (eis) }\end{array}$ & $\begin{array}{c}\text { Pedreiro (Pai) } \\
\text { e } \\
\text { Serviços } \\
\text { gerais (Mãe) }\end{array}$ & $\begin{array}{c}\text { Trabalha em } \\
\text { oficina }\end{array}$ & Cozinheira & $\begin{array}{l}\text { Trabalha } \\
\text { com } \\
\text { mudanças } \\
\text { (Pai) } \\
\text { e } \\
\text { Faxineira } \\
\text { (Mãe) }\end{array}$ & $\begin{array}{l}\text { Auxiliar de } \\
\text { Limpeza }\end{array}$ & Diarista \\
\hline
\end{tabular}

\section{Delineamento do estudo e Procedimentos}

$\mathrm{O}$ presente estudo tem um delineamento qualitativo. Como Vasmoirade, Turunen e Bondas (2013) apontaram, as abordagens qualitativas compartilham um objetivo semelhante, na medida em que buscam chegar à compreensão de um determinado fenômeno da Barbarói, Santa Cruz do Sul, n. 58, p.<47-64>, jan/jun 2021 
perspectiva de quem o vivencia. Da mesma forma, o delineamento qualitativo consiste em várias abordagens para a coleta de dados, que os pesquisadores podem usar para ajudar ao prover uma descrição cultural e contextual e uma interpretação de fenômenos sociais (VASMOIRADE; SNELGROVE, 2019).

O presente estudo foi aprovado pelo Comitê de Ética em Pesquisa da Universidade e pela Secretaria de Saúde do município em questão. Para a realização dessa pesquisa contou-se com a mediação inicial de agentes comunitárias de saúde de duas unidades de Estratégia da Saúde da Família de um município no interior do Rio Grande do Sul, os quais indicavam famílias com irmãos cuidadores. A proposta da pesquisa foi apresentada formalmente aos responsáveis legais dos participantes, quando estes eram menores de idade, e somente após seu consentimento escrito foi iniciado o contato com os adolescentes. Do mesmo modo, foi explicitado aos participantes questões relativas à pesquisa e seus objetivos, e assim, foi obtido seu assentimento escrito em participar dessa atividade. $O$ termo de consentimento livre e esclarecido também foi disponibilizado quando os adolescentes eram maiores de idade.

A coleta das informações foi realizada por meio de entrevistas semiestruturadas na casa dos adolescentes. Seguiu-se um roteiro prévio de entrevista, o qual abrangeu dados sociodemográficos, informações sobre a família do participante, sobre a sua rotina de atividades, assim como sua percepção sobre a experiência de cuidar de irmãos.

\section{Análise dos Dados}

Todas as entrevistas foram gravadas e realizadas na casa dos participantes, e foram posteriormente transcritas e submetidas à análise de conteúdo. Este tipo de análise caracterizase por "uma abordagem sistemática de codificação e categorização usada para explorar grandes quantidades de informações textuais discretamente para determinar tendências e padrões de palavras usadas, sua frequência, suas relações e as estruturas e discursos de comunicação" (VASMOIRADE et al., 2013, p. 3).

\section{Resultados e Discussões}

A partir da análise da caracterização da amostra e do levantamento dos dados referentes à família dos participantes desta pesquisa, é possível perceber que a maioria dos participantes desta pesquisa é do sexo feminino. Além disso, 4 das 6 famílias se constituem como monoparentais, entre estas, apenas uma era um homem era o chefe da família, e em três, 
mulheres. O número de pessoas da família que moram na mesma casa variou amplamente, de 4 a 9 pessoas, com média de 6,3 moradores na mesma casa.

No mesmo sentido das características encontradas neste estudo, considera-se que alguns fatores parecem estar mais relacionados à constituição da situação de cuidado entre os irmãos, dentre eles estão a configuração familiar monoparental, o elevado número de filhos e membros da família, e baixo nível socioeconômico da família (DELLAZZANA, 2008; POLETTO; WAGNER; KOLLER, 2004; WEBBINK; SMITS; DE JONG, 2012). Aspectos relacionados às questões de gênero também se destacam, pois são as meninas que realizam grande número de atividades de cuidado com os irmãos mais novos, bem como atividades domésticas (DELLAZZANA, 2008; FRENCH, 2010; PLAN INTERNATIONAL BRASIL, 2014; SILVA; TOKUMARU, 2008). Os resultados e a análise das informações serão apresentados em duas categorias: "Atividades cotidianas de cuidado aos irmãos" e "Percepções das práticas de cuidado aos irmãos", as quais emergiram das entrevistas.

\section{Atividades cotidianas de cuidado dos irmãos}

Esta categoria descreve a caracterização das atividades realizadas pelos adolescentes cuidadores deste estudo. Em alguns contextos familiares, a delegação de responsabilidades e tarefas para os filhos é entendida como em favor da sobrevivência do próprio grupo familiar, sendo a participação dos irmãos mais velhos em atividades domésticas e de cuidado considerada essencial para a manutenção financeira da família (DELLAZZANA, 2008; DELLAZZANA-ZANON; FREITAS, 2010; 2012). Dessa forma, é possível verificar a partir das falas dos participantes que as atividades rotineiras de cuidado dispensadas aos seus irmãos começam quando os pais, as mães, os irmãos mais velhos e o padrasto saem para o trabalho. Tal como apresentado nos relatos de P1 e P4:

Enquanto os outros saem para trabalhar fico com $N$ (P1, feminino, 18 anos).

A mãe vai trabalhar eu cuido dos meus irmãos (P4, masculino, 14 anos).

Assim, ressalta-se a importância das atividades desempenhadas pelos adolescentes no âmbito doméstico para o sustento da família. No que diz respeito à avaliação das diversas implicações nas tarefas de cuidado, é importante considerar as características das atividades as quais os adolescentes assumem. Algumas pesquisas abordam contextos familiares da população em geral, nos quais há níveis "normais" de provisão de assistência pelos 
adolescentes à sua família e os associam ao bem-estar proporcionado a eles, exemplo, altos níveis de felicidade devido a sua percepção de estar cumprindo um papel importante na família (TELZER; FULIGNI, 2009). Outras avaliam situações em que o adolescente assume extensivamente papéis de adultos, ocasionando uma carga excessiva de atividades que excedem a capacidade do adolescente de lidar com elas de forma saudável (BURTON, 2007) e prejudicam o seu desempenho escolar (DELLAZZANA-ZANON; FREITAS, 2010). As atividades de três participantes foram bastante parecidas devido à idade dos irmãos mais novos ( 2 anos; 8 meses e 3 anos; 6 meses), os quais ainda requerem cuidados bem específicos como maior supervisões dos comportamentos da criança, cuidar da higiene e saúde dos irmãos.

Eu faço mamá pro P., faço ele dormir, eu mudo ele, dou janta pra eles, pro G. e pra ele (P3, feminino, 14 anos).

Ah aprendi a cuidar de nenê, dar mamá, fazer dormir e trocar as fraldas. Eu sei fazer tudo isso aí..." (P4, masculino, 14 anos).

Banho, alimentar ele né, é isso, quando tá com dor eu dou remedinho pra ele, ai ele para, mas ele nem incomoda (P 5, feminino, 19 anos).

Destaca-se, assim, a grande responsabilidade dos participantes no exercício do cuidado, o que pode gerar reconhecimento pelos familiares, mas também, uma maior sobrecarga de atividades. Quando os irmãos apresentam idade um pouco mais elevada, outros cuidados são assumidos, como buscar na escola, ajudar nas tarefas escolares, ministrar remédios, administrar a divisão de tarefas domésticas e mediar conflitos entre eles.

Eu tenho que fazer os temas com eles e quando eles sentam no sofá tem um de um lado e outro do outro lado ai a gente explica uma coisa pra um e outra coisa pro outro, é um monte (P6, masculino, 17 anos).

Mais preocupante é os pequenos porque dai, aquela ali tem que fazer bombinha e a $\mathrm{N}$. também, ai são três vezes ao dia, a N. se acorda de manhã eu faço bombinha, ai de tarde, a hora que ela chega do colégio eu tenho que fazer de novo, antes de dormir eu tenho que fazer de novo, ai de manhã eu tenho que dar remédio antialérgico pra elas, ai de meio-dia antes delas almoçarem tenho que dar Prednisolona pras duas, e as vezes quando elas tão com tosse eu tenho que fazer nebulização três vezes por dia também (P6, masculino, 17 anos). 
Tem vezes que teima: não, eu não vou fazer isso, eu não quero fazer isso. E depois vou la e converso, converso, converso e quando eu to quase quatro horas conversando eles dizem: ta, eu faço mas é só isso (P1, feminino, 18 anos).

Neste estudo, a caracterização das atividades de cuidado foi bastante diversificada para cada participante. Segundo Evans (2012) o papel de cuidado exercido por jovens pode ser maior ou menor, no que depende, dentre outros fatores, da necessidade de cuidado de cada membro da família e da disponibilidade de outros cuidadores para o desempenho dessas atividades. MacMahon e Luttar (2007), por sua vez, enfatizam que a questão das repercussões dessas tarefas em diversos aspectos da vida do cuidador não está nos níveis de envolvimento no cuidado oferecido, porém, está mais relacionado ao contexto onde o cuidado ocorre, como ambiente familiar não responsivo e que oferece pouco apoio. Portanto, é importante apontar o papel da família como suporte nos momentos de dificuldade na rotina de cuidados dos adolescentes, bem como, reforçar a necessidade de maior supervisão e cuidados dispensados a bebês e crianças pequenas. Os adolescentes têm direito resguardado no que se refere ao lazer e também ao bom aproveitamento e desempenho educacional. Salienta-se que neste estudo os participantes em sua maioria possuem ensino fundamental ou médio incompletos, algo que deve ser destacado visto a necessidade de maior atenção ao desenvolvimento escolar desses adolescentes, bem como, às atividades que desempenham.

\section{Percepções das práticas de cuidado dos irmãos}

Esta categoria aborda as percepções dos adolescentes em relação às suas práticas de cuidado com irmãos. Em muitos casos, o cuidado está intrincado em responsabilidade e obrigações familiares e é, culturalmente, direcionado às mulheres. Dessa forma, as tarefas são percebidas como "normal", "rotina", "tarefas de todo dia". Em razão de essas atividades serem realizadas dentro de casa e destinadas a indivíduos da própria família contribuem para serem naturalizadas até mesmo pelos jovens cuidadores, vistas como normal na relação familiar (SMYTH; BLAXLAND; CASS, 2011), como observam a P1 e P5.

Olha, pra mim é uma coisa normal, não é assim como uma grande, grande responsabilidade (P1, feminino, 18 anos).

Já virou rotina já [...] mas é bom cuidar deles, eles nem incomodam (P5, feminino, 19 anos). 
Duas participantes consideraram exercer o papel de mãe para seu irmão e P6 reconhece a sua importante contribuição na educação dos irmãos. Segundo Poletto, Wagner e Koller (2004), as meninas que cuidam de seus irmãos podem aprender a cuidar, reconhecer as reações e as necessidades de seus irmãos, fornecendo a eles cuidados maternais.

É uma segunda mãe, o P. me chamava de mãe (P3, feminino, 14 anos).

O M. parece o meu filho (risos) que desde pequenininho eu cuido ele né, bem novinho (P5, feminino, 19 anos).

Eu faço de tudo por eles, tudo, tudo, aquela ali eu ensinei a falar, ensinei tudo, e tudo que ela [mãe] não ensinou eu ensinei pra eles, tudo que eles sabem (P6, masculino, 17 anos).

Portanto, a percepção dos participantes em relação às funções que assumem, pode contribuir para a sensação de importância no contexto familiar, como também pode ocasionar um sentimento de falta da presença das mães e dos pais nas responsabilidades que deveriam ser por eles realizadas. Ser identificado como "cuidador" pelos outros pode ser motivo de orgulho, de reconhecimento da responsabilidade que eles carregam dentro da família (SMYTH; BLAXLAND; CASS, 2011). Nesse sentido, Dahlblom et al. (2009) apontam que os cuidadores percebem de forma predominantemente positiva as suas atividades de cuidado, muitas vezes demonstrando estarem orgulhosos com os cuidados que realizavam. Telzer e Fuligni (2009) indicam que a assistência diária realizada por adolescentes às suas famílias esteve associada com altos níveis de felicidade devido, em parte, pela percepção de cumprimento de um papel importante na família. Aspectos que podem ser observados nas falas dos participantes e aqui representada pelo relato de P1:

É bom assim sabe, começa assim a ter convivência com o irmão mais novo, [...] é bom saber que está transmitindo algo de bom pra outra pessoa (P1, feminino, 18 anos).

Nos casos em que adolescentes do sexo masculino são quem provêm o cuidado, é possível que haja resistências ao falarem sobre suas atividades ou se autodenominarem cuidadores em face às expectativas sociais sobre a masculinidade (SMYTH; BLAXLAND; CASS, 2011). Em contrapartida, o P4 parece sentir orgulho em dizer que sabe realizar atividades de cuidado como dar mamadeira e fazer dormir, relata gostar da sua rotina de 
cuidados prestados aos seus irmãos e que encontra facilidade nas atividades que foram ensinadas pela mãe ou pelo tio.

Eu acho legal até porque eu aprendi com 8 anos, dai é fácil. [...] Ah, aprendi a pegar ele no colo, dar mamá e fazer dormir foi fácil porque a mãe me ensinou tudo se não os meus tios [...] Tem amigo meu que cuida de irmão quando as mãe saem, tipo eu, mas eu gosto (P4, masculino, 14 anos).

Alguns fatores estão ligados aos impactos psicológicos positivos dessa prática de cuidado. Os cuidadores podem desenvolver uma percepção de pertencimento e responsabilidade dentro do grupo familiar e, quando percebem que desempenham bem as atividades de cuidado aos irmãos mais novos, eles podem desenvolver a sensação de dever cumprido (YANAGIZAWA; POUDEL; JIMBA, 2010). Dessa forma, ao encontrar facilidade nas tarefas, o P4 gosta de desempenhá-las. Destaca-se a idade precoce com que aprendeu as tarefas relativas a cuidado dos irmãos.

Entretanto, nem todos os participantes avaliaram sua experiência de cuidar de irmãos como positiva. Para um dos participantes (P2), o cuidado dispensado é difícil por ainda não possuir o exercício da autoridade e se vê impelida a resolver problemas de brigas entre seus irmãos, por isso, ela mesma é castigada pelo pai.

[..] eles incomodam muito. [...] os dois brigam, fazem bagunça [...] Eu não posso me meter, depois sobra tudo pra mim (P2, feminino, 11 anos).

O estudo realizado por Dellazzana-Zanon e Freitas (2012) com 16 adolescentes com idade entre 12 e 16 anos, com objetivo de examinar os tipos de sanção escolhidos por adolescentes de nível socioeconômico baixo, que cuidam e educam regularmente seus irmãos, concluiu que o uso da reciprocidade do diálogo para mediar os conflitos entre os irmãos. Alguns adolescentes podem encontrar dificuldades de impor limite aos comportamentos dos irmãos, algo que é da responsabilidade das figuras parentais.

Em outra perspectiva, a P3 relatou muita tristeza pela sua rotina, visto que é desgastante e que a priva de momentos de lazer. Já P6 refere sua rotina como estressante. A fala destes adolescentes revela a tristeza e estresse em relação à obrigação no desempenho dessas atividades. 
É que tipo assim, eu sou nova né, eu deveria estar aproveitando a vida do que cuidando de irmão (choro) (P3, feminino, 14 anos).

Ah é muito estressante. É muito berro, é muito grito que eu tenho que dar com eles pra eles me ouvirem, é horrível (P6, masculino, 17 anos).

A prática de cuidado pode relacionar-se a implicações psicológicas negativas, que incluem estresse, exaustão emocional e tempo para diversão limitado (YANAGIZAWA; POUDEL; JIMBA, 2010). Na mesma direção, Dellazzana (2008) e Dellazzana-Zanon e Freitas (2010) apontam que o tempo escasso para atividades de lazer se encontra relacionado com as tarefas assumidas no âmbito doméstico. A falta de tempo na rotina do cuidador causalhe cansaço e fadiga e implica negativamente na sua saúde e resultados escolares (Evans, 2012). Níveis aumentados de depressão estiveram significativamente associados com a tarefa de cuidado exercida por adolescentes (YI et al., 2012). A alta carga emocional na rotina do jovem e o possível estresse decorrente disso foi mencionado por P6.

Embora o cuidado dos irmãos desperte sentimentos ambivalentes nos participantes desse estudo, bem como, gerar estresse e desgaste, os adolescentes referem ter apoio dos familiares, tal como retrata $\mathrm{P} 4$ :

No primeiro dia eu dei um colo nele, foi difícil [...] eu tinha medo de pegar né, não sabia dar colo, com medo de afogar ele. E a mãe me ensinou (P4, masculino, 14 anos)

Pais e mães orientaram os adolescentes para a realização de tarefas como dar comida e cuidar dos irmãos e assistir televisão. Em alguns momentos, os adolescentes referiram utilizar métodos punitivos para controlar o comportamento dos irmãos, como agressão física. Em momentos de tensão e estresse extremo, P6 revelou o uso da agressão física como último recurso para que os irmãos pudessem obedecê-lo.

Um dá tapa no outro, um dá tapa no outro, aí tu tem que botar um em cada ponta, aí quando tu bota eles começam a te xingar por ter se separado, aí o cara tem que pensar cinco ou seis vezes para não bater [. ..] Bati porque falei, falei e eles não me ouviram e só abaixo de pau para eles me ouvirem (P6, masculino, 17 anos). 
Desentendimentos, rivalidades e conflitos podem ser entendidos como eventos presentes na relação fraterna como um laboratório de experiências relacionais, em função do estágio de desenvolvimento em que os irmãos se encontram (POLETTO; KOLLER; DELL'ÁGLIO, 2009). Porém, estudos analisaram os eventos estressantes percebidos pelos adolescentes e constataram que "brigas com irmãos" era um evento frequente e considerado de maior impacto para crianças e adolescentes que viviam com sua família (MORAES; KOLLER; RAFFAELli, 2010; POLETTO; KOLLER; DELL'ÁGLIO, 2009). Portanto, é imprescindível considerar o tipo de discussão ou briga em que os irmãos se envolvem, uma vez que a violência tem mostrado implicações negativas para a saúde mental de crianças e jovens (TUCKER et al., 2013). Portanto, mais atenção é necessária para eventos como esses, pois episódios crônicos de violência entre irmãos podem indicar efeitos adversos em crianças e adolescentes (BUTTON; GEALT, 2010).

Nesse sentido, Cohen et al. (2012) ressaltam a atenção que deve ser dada à maneira como os adolescentes cuidadores adquirem habilidades na execução das tarefas e como eles lidam com os acertos e os erros neste processo de aprendizagem. Desse modo, é importante evidenciar as implicações negativas que essas atividades podem ter, já que a responsabilidade em assumir o cuidado de irmãos mais novos pode gerar impacto negativo para a saúde mental e bem-estar dos adolescentes e crianças (FRENCH, 2010). Para avaliar as implicações dessas práticas cotidianas para os adolescentes muitas variáveis precisam ser consideradas como as expectativas culturais quanto ao sexo, idade, os conceitos de família, de responsabilidade por parte dos filhos e a qualidade das interações entre irmãos (HAFFORD, 2010). A realização de estudos longitudinais pode trazer grandes contribuições para o estudo das consequências dos cuidados formais para o desenvolvimento tanto dos adolescentes cuidadores quanto daqueles que são cuidados (EAST, 2010).

\section{Conclusões}

O presente estudo buscou investigar as perspectivas de adolescentes sobre a prática de cuidado dos irmãos. Primeiramente, foi possível notar a interação do cuidador de irmãos (microssistema), aspecto do macrossistema e suas relações com a teoria bioecológica de Bronfenbrenner, uma vez que a maioria das famílias caracterizou-se como monoparental, apresentando elevado número de filhos e familiares, bem como de camadas populares. Além disso, também foi possível notar as questões de gênero no cuidado de irmãos, considerando que a maioria dos irmãos cuidadores era do sexo feminino, o que denota a manutenção do 
lugar de cuidado como uma função das mulheres, algo observado e perpetuado na sociedade brasileira.

Em segundo lugar, foi possível perceber diversos aspectos que estão relacionados à prática de cuidar dos irmãos, que se estabelece como importante para a organização e suporte do microssistema familiar. A percepção da prática do cuidado como algo "normal" e parte do “cotidiano" esteve presente neste estudo, o que evidencia a naturalização da prática até mesmo pelos cuidadores. O cuidado, então, foi visto como um auxílio à família.

Aspectos positivos e negativos também foram relatados pelos participantes. As percepções negativas referiram-se à limitação dos tempos de lazer, à rotina estressante e aos momentos de mediação das brigas entre os irmãos menores. Além do atraso escolar, bem expressivo nesta amostra pesquisada. A tristeza e o descontentamento com a rotina de cuidar dos irmãos estiveram presentes na fala de três participantes deste estudo. Nestes casos, a atividade de cuidado está associada a sentimentos de desconforto, que podem influenciar no bem-estar desses adolescentes.

Aqueles que perceberam suas tarefas como positivas encontraram aspectos como a diversão em conjunto, a sensação de estar transmitindo algo de bom para eles. Os desafios e facilidades para realizar atividades de cuidado com os irmãos mais novos pode contribuir para o sentimento de reconhecimento em relação ao seu desempenho, tendo um papel importante na família, mas também pode gerar ressentimento pela falta de responsabilidades das figuras parentais com o cuidado dos filhos.

A situação de cuidado entre irmãos é comumente estabelecida em várias famílias e, portanto, destaca-se a necessidade de analisar o que isso representa para os adolescentes cuidadores e como se organiza junto às demais atividades cotidianas. Explorar as atividades desempenhadas pelo adolescente cuidador, as implicações para diversos aspectos de sua vida, bem como, suas perspectivas sobre essa prática é relevante por permitir maior visibilidade dessa prática além de possibilitar que os profissionais que atuam com famílias atentem para este fenômeno e realizem ações orientadoras.

Além disso, estudos nessa linha permitem que se tensione o Estado e órgãos governamentais a fim de cumprir a garantia de direitos estabelecidos no Estatuto da Criança e do Adolescente (Estatuto da Criança e do Adolescente [ECA]; BRASIL, 2007). Nesta perspectiva, é importante destacar que o ECA garante a criança e ao adolescente todos os direitos fundamentais, de modo a possibilitar o desenvolvimento físico, moral, mental, social, espiritual, dignidade e liberdade (BRASIL, 2007). Ademais, este Estatuto ressalta que tanto 
crianças como adolescentes não deverão ser expostos a quaisquer formas de negligência, exploração, discriminação, crueldade, opressão e discriminação. O ECA prevê ainda que é proibido as pessoas com idade menor de quatorze anos, o desempenho de qualquer forma de trabalho, salvo aqueles na condição de aprendiz. O presente estudo destaca também a possibilidade de reflexão sobre a disponibilidade de vagas em creches e escolas de ensino infantil públicas como importante recurso para apoiar as famílias no cuidado dos filhos pequenos e, assim, permitir que os adolescentes invistam na sua educação escolar e apresentem maior tempo livre para o lazer.

Por fim, destaca-se que o presente estudo não pretende generalizar os dados, mas sim abordar as atividades e percepções dos adolescentes sobre o cuidado aos irmãos. As limitações do estudo referem-se ao número reduzido de participantes da amostra. Ressalta-se a necessidade de realização de pesquisas sobre o cuidado entre irmãos em diferentes perspectivas e contextos, atualmente escassos, para que a construção do conhecimento nesta área possa se desenvolver. Como sugestão para pesquisas futuras, seria relevante incluir outros participantes, avaliando as perspectivas de pais, mães dos cuidadores e dos irmãos sob seus cuidados.

\title{
PERSPECTIVES OF ADOLESCENTS ABOUT THEIR SIBLING CARETAKING: A QUALITATIVE STUDY
}

\begin{abstract}
This study sought to investigate the perspectives of adolescents about their sibling caretaking. The sample consisted of six adolescents of both sexes, aged between 11 and 19 years old. The data were collected through semi-structured interviews and were submitted to content analysis. The importance of the activities performed by adolescents in the family context for the financial support of the family is emphasized. The caregiver's perception of the care given to siblings contained positive and negative aspects, and was also considered to be routine practice. Among the positives factors, it was found to transmit something good to the siblings, the challenges and facilities in carrying out care activities to younger siblings. The sibling caretaking practice can contribute to the feeling of importance in the family context, as well as being perceived as a failure of mothers in carrying out their responsibilities. Negative perceptions referred to the limitation of leisure time, the stressful routine and the mediation of fights between younger siblings. The importance of analyzing what this practice represents for young caregivers is evident, as it can be associated with uncomfortable feelings, influence well-being, as well as limit the performance of leisure activities.
\end{abstract}

Keywords: Siblings; Caregivers; Adolescent. 


\section{PERSPECTIVAS DE LOS ADOLESCENTES SOBRE LA PRÁCTICA DE CUIDADO DEL HERMANO: UN ESTUDIO CUALITATIVO}

\section{Resumén}

Este estudio buscó investigar las perspectivas de los adolescentes sobre la práctica del cuidado de los hermanos. La muestra estuvo formada por seis adolescentes de ambos sexos, con edades comprendidas entre los 11 y los 19 años. La información se recopiló a través de entrevistas semiestructuradas y la información se sometió a análisis de contenido. Se enfatiza la importancia de las actividades que realizan los adolescentes en el contexto familiar para el apoyo financiero de la familia. La percepción del cuidador sobre el cuidado brindado a los hermanos contenía aspectos tanto positivos como negativos, y también se considera una práctica normal y rutinaria. Entre los puntos positivos, se encontró transmitir algo bueno a los hermanos, los desafíos y facilidades para realizar actividades de cuidado con los hermanos menores. La práctica del cuidado puede contribuir al sentimiento de importancia en el contexto familiar, además de ser percibida como un fracaso de las madres en el cumplimiento de sus responsabilidades. Las percepciones negativas se referían a la limitación del tiempo libre, la rutina estresante y la mediación de peleas entre hermanos menores. Es evidente la importancia de analizar lo que representa esta práctica para los cuidadores adolescentes, ya que puede asociarse a sentimientos de malestar, influir en el bienestar, así como limitar la realización de actividades de ocio.

Palabras Clave: Hermanos; Cuidadores; Adolescente.

\section{REFERENCIAS}

BURTON, L. Childhood Adultification in Economically Disadvantaged Families: A

Conceptual Model. Family Relations, v. 56, p. 329-345, out. 2007.

BUTTON, D. M., GEALT, R. High Risk Behaviors Among Victims of Sibling Violence. Journal of Family Violence, v. 25, 131-140, 2010.

BRASIL, Ministério da Saúde. Estatuto da Criança e do Adolescente. 3 ed. Brasília: Editora do Ministério da Saúde. Série E. Legislação de Saúde, 96p, 2007.

BRONFENBRENNER, U. The ecology of human development: Experiments by nature and design. Cambridge, MA: Harvard University Press, 1979.

COHEN, D. et al. Impact of family caregiving by youth on their psychological well-being: A latent trait analysis. Journal of Behavioral Health Services \& Research, v. 39, n.3, p. 245-256, 2012.

DALHBLOM, K. et al. Home Alone: Children as Caretakers in León, Nicaragua. Children \& Society, v. 23, p. 43-56, 2009.

DELLAZZANA, L. L. Irmãos que cuidam de irmãos: Concepções de justiça retributiva. 2008. 121 f. Dissertação (Mestrado em Psicologia do Desenvolvimento) - Universidade Federal do Rio Grande do Sul, Porto Alegre, 2008.

DELLAZZANA-ZANON, L. L.; FREITAS, L. B. L. Um dia na vida de irmãos que cuidam de irmãos. Psicologia: Teoria e Pesquisa, v.26, n. 4, pp.595-603., 2010. 
DELLAZZANA-ZANON, L. L.; FREITAS, L. B. L. Tipos de sanção escolhidos por adolescentes que cuidam e educam seus irmãos menores. Nuances: estudos sobre Educação, Ano XVIII, v. 23, n. 24, p. 78-94, set./dez. 2012

EAST, P. Children's Provision of Family Caregiving: Benefit or Burden? Child Development Perspective, v. 4, n.1, p. 1-10, abr. 2010.

EVANS, R. Sibling caringscapes: Time-space practices of caring without youth-headed households in Tanzania and Uganda. Geoforum, v.43, p. 824-835, 2012.

FRENCH, J. L. Children's Labor Market Involvement, Household Work, and Welfare: A Brazilian Case Study. Journal of Business Ethics, v. 92, p. 63-78, 2010.

HAFFORD, C. Sibling caretaking in immigrant families: Understanding cultural practices to inform child welfare practice and evaluation. Evaluation and Program Planning, v. 33, p. 294-302, 2010.

LAM, C. B., MCHALE, S. M., CROUTER, A. C. Parent-Child Shared Time From Middle Childhood to Late Adolescence: Developmental Course and Adjustment Correlates. Child Development,v. 83, n. 6, p. 2089-2103, 2012.

MACMAHON, T. J.; LUTHAR, S. S. Defining Characteristics and Potential Consequences of Caretaking Burden Among Children Living in Urban Poverty. American Journal of Orthopsychiatry, v. 77, n. 2, p. 267-281, 2007.

MERMELSHTINE, R. Parent-child learning interactions: A review of the literature on scaffolding. British Journal of Educational Psychology, v. 87, n. 2, p. 241-254, 2017.

MORAES, N. A., KOLLER, S. H., RAFFAELLI, M. Eventos estressores e indicadores de ajustamento entre adolescentes em situação de vulnerabilidade social no Brasil. Universitas Psychologica, v. 9, n. 3, p. 787-806, 2010.

ONWUEGBUZIE, A. J., COLLINS, K. M. T., FRELS, R. K. Foreword: Using Bronfenbrenner's ecological systems theory to frame quantitative, qualitative, and mixed research. International Journal of Multiple Research Approaches, v. 7, n. 1, p. 2-8, 2013.

PEREIRA, C. R. R., PICCININI, C. A., LOPES, R. C. S. (2019). Relacionamento fraterno na infância: Uma compreensão sistêmica do desenvolvimento. In. DESSEN, M. A. (Org.). Família no curso de vida: compreendendo a família e seus desafios na contemporaneidade. Curitiba: Juruá, v.1, 2019.

POLETTO, M., KOLLER, S. H., DELL'ÁGLIIO, D. D. Eventos estressores em crianças e adolescentes em situação de vulnerabilidade social de Porto Alegre. Ciência \& Saúde Coletiva, v. 14, n. 2, p. 455-466, 2009.

POLETTO, M., WAGNER, T. M. C, KOLLER, S. H. Resiliência e Desenvolvimento Infantil de crianças que cuidam de crianças: Uma visão em perspectiva. Psicologia: Teoria e Pesquisa, v. 20, n.3, p. 241-250, 2004. 
PLAN INTERNATIONAL BRASIL. Resumo Executivo. Brasília: Plan International Brasil, 2014.

RUIZ-CASARES, M.; HEYMANN, J. Children home alone unsupervised: Modeling parental decisions and associated factors in Botswana, México, and Vietnam. Child Abuse \& Neglect, v. 33, p. 312-323, 2009.

SILVA; L. W. S.; TOKUMARU, R. Cuidados Parentais e Aloparentais Recebidos por Crianças de Escolas Públicas e Particulares de Vitória - ES. Psicologia: Reflexão e Crítica, v. 21, n. 1, p.133-141, 2008.

SMYTH, C; BRAXLAND, M.; CASS, B. 'So that's how I found out I was a young carer and that I actually had been a carer most of my life': Identifying and supporting hidden young carers. Journal of Youth Studies, v. 14, n. 2, p. 145-160, 2011.

TELZER E. H; FULIGNI A. J. Daily Family Assistance and the Psychological Well-Being of Adolescents From Latin American, Asian, and European Backgrounds. Developmental Psychology, v. 45, n. 4, p. 1177-1189, 2009.

TUCKER, C. J. et al. Association of Sibling Aggression With Child and Adolescent Mental Health. Pediatrics, v. 132, n. 1, p. 79-84, 2013.

TUCKER, C. J., UPDEGRAFF, K. The relative contributions of parents and siblings to child and adolescent development. New Directions for Child and Adolescent Development, $n$. 126, p. 13-28, 2009.

VAISMORADI, M., TURUNEN, H., BONDAS, T. Content analysis and thematic analysis: Implications for conducting a qualitative descriptive study. Nursing \& Health Sciences, v. 15, n. 3, p. $1-8,2013$.

VAISMORADE, M., SNELGROVE, S. Theme in Qualitative Content Analysis and Thematic Analysis. Forum Qualitative Sozialforschung/Forum: Qualititative social research, v. 29, $n$. 3, Art. 23, 2019.

YANAGIZAWA, S.; POUDEL, K. C.; JIMBA, M. Sibling caregiving among children orphaned by AIDS: Synthesis of recent studies for policy implications. Health Policy, v. 98, p. 121-130, 2010.

YI, S. et al. Sibling care, school performance, and depression among adolescent caretakers in Cambodia. Asian Journal of Psychiatry, v. 5, p. 132-136, 2012.

WEBBINK, E., SMITS, J., de JONG, E. Hidden Child Labor: Determinants of Housework and Family Business Work of Children in 16 Developing Countries. World Development, v. 40, n. 3, p. 631-642, 2012.

WEISNER, T. S., GALLIMARE, R. My brother's keeper: Child and sibling caretaking. Current Anthropology, v. 18, n. 2, p. 169-180, 1977. 
WIKLE, J. S., JENSEN, A. C., HOAGLAND, A. M. Adolescent caretaking of younger siblings. Social Science Research, v. 71, p. 72-84, 2018.

Data de recebimento: 30/09/2015

Data de aceite: $16 / 11 / 2020$

\section{Sobre as autoras:}

Gabriella Koltermann é Psicóloga graduada pela Universidade Federal de Santa Maria (UFSM). Mestre e Doutoranda em Psicologia pela Universidade Federal do Rio Grande do Sul (UFRGS), Porto Alegre, RS, Brasil. Endereço Eletrônico: gabikoltermann@ gmail.com

Meiridiane Domingues de Deus é Graduada em Psicologia pela Universidade Federal de Santa Maria - UFSM. Especialista em Gestão de Organização Pública em Saúde e Mestra em Psicologia pela UFSM. Especialista em Design Instrucional. Doutora em Psicologia pela Universidade Federal de Santa Catarina (UFSC), Florianópolis, SC, Brasil. Endereço Eletrônico: meiridomingues@ hotmail.com

Samara Silva dos Santos é Doutora em Psicologia pela Universidade Federal do Rio Grande do Sul (UFRGS). Professora Adjunta do Departamento de Psicologia e do Programa de PósGraduação em Psicologia da Universidade Federal de Santa Maria (UFSM), Santa Maria, RS, Brasil. Endereço Eletrônico: silvadossantos.samara@gmail.com 